ÉGYPTE monde arabe

\section{Égypte/Monde arabe}

4 | 1990

Démocratie et démocratisation dans le monde arabe

\title{
Les élections étudiantes en Cisjordanie (1978-1987)
}

Jean-François Legrain

\section{OpenEdition}

\section{Journals}

Édition électronique

URL : https://journals.openedition.org/ema/345

DOI : 10.4000/ema.345

ISSN : 2090-7273

Éditeur

CEDEJ - Centre d'études et de documentation économiques juridiques et sociales

Édition imprimée

Date de publication : 31 décembre 1990

ISSN : 1110-5097

Référence électronique

Jean-François Legrain, « Les élections étudiantes en Cisjordanie (1978-1987) », Égypte/Monde arabe [En ligne], 4 | 1990, mis en ligne le 08 juillet 2008, consulté le 07 juillet 2022. URL : http:// journals.openedition.org/ema/345; DOI : https://doi.org/10.4000/ema.345

Ce document a été généré automatiquement le 7 juillet 2022

Tous droits réservés 


\title{
Les élections étudiantes en Cisjordanie (1978-1987)
}

\author{
Jean-François Legrain
}

1 Depuis 1967, l'émergence, le renforcement ou l'affaiblissement des leaderships en Cisjordanie et dans la Bande de Gaza se trouvent pris entre la population locale première concernée, Israël puissance occupante, la Jordanie puissance nominalement souveraine jusqu'en juillet 1988 et l'Organisation de Libération de la Palestine (OLP) reconnue « unique représentant légitime du peuple palestinien » par le Sommet arabe de Rabat en 1974'. Depuis la fin 1987, avec le soulèvement (Intifâda), I'« occupation normalisée » des vingt années précédentes se trouve radicalement ébranlée : tandis qu'une bi-polarité se met en place entre les nationalistes partisans de l'OLP et les Frères musulmans du Mouvement de la Résistance Islamique, "l'Intérieur» arrache l'initiative politique à « l'Extérieur $»^{2} ; l^{\prime}$ observateur est ainsi conduit à s'interroger sur la possible marginalisation de personnalités jusque-là considérées comme les leaders locaux à destin national ainsi que sur l'identité d'éventuels nouveaux acteurs sur la scène sociale et politique.

2 En d'absence de toute élection "nationale " (les dernières élections organisées sur l'ensemble de la Cisjordanie, à l'exception de Jérusalem-Est annexée, remontent à 1976 et ont eu lieu à l'échelon municipal), il revient à l'observateur d'identifier ou de construire des échantillons suffisamment représentatifs pour donner, à chaque période importante, une représentation adéquate du leadership et de son mode de fonctionnement. Lors du précédent colloque franco-égyptien de politologie ${ }^{3}, j^{\prime}$ ai tenté de dessiner les contours de ce leadership en analysant la composition des structures clandestines et semi-clandestines mises en place depuis 1967 (Fronts patriotiques et Comités d'Orientation Nationale) et les représentations de la scène politique que se sont donnés différents acteurs reconnus ("Charte des Arabes ", "Document Husaynî », Invités des Chancelleries). J'avais ainsi montré le processus de mise à l'écart des notables liés à la Jordanie au profit de nouveaux leaders rattachés aux organisations de l'OLP ainsi que celui de la montée des islamistes jamais reconnue par les nationalistes; la sociologie de ces leaders faisait apparaittre la sous-représentation chronique des 
femmes et des ruraux, la mise à l'écart de facto des réfugiés (des camps comme hors les camps), la surreprésentation des chrétiens, le processus de concentration des leaders dans la conurbation de Jérusalem au détriment de la Bande de Gaza comme des autres anciens pôles urbains de Cisjordanie (Naplouse et Hébron) et la marginalisation croissante des milieux économiques (propriétaires terriens, industriels et hommes d'affaires) au profit des « intellectuels » (enseignants du supérieur et journalistes).

3 Les élections aux conseils étudiants des diverses universités de Cisjordanie offrent un échantillon de choix du leadership de "l'Intérieur», certes limité à une catégorie professionnelle particulière et à une classe d'âge restreinte, mais intensément engagé dans la lutte politique. Ces élections peuvent être considérées comme démocratiques : la liberté de candidature est totale; le scrutin est secret; surveillé par un comité d'arbitrage constitué de membres des administrations et du corps enseignant ainsi que par des observateurs issus des différentes listes en lice, il ne peut être l'objet de manipulations dans le comput des voix; ces élections, enfin, se traduisent par un renouvellement des représentants étudiants et par d'éventuels changements dans l'organisation de la vie universitaire en cas de renversement de tendances. Il va sans dire que cette possibilité de tenir des élections démocratiques, que l'on retrouve dans le milieu associatif, professionnel et caritatif, ne signifie pas que l'occupation israélienne est une "occupation démocratique »: le droit à l'autodétermination, fondement et fin de toute démocratie, est officiellement dénié. L'occupation, cependant, dans le cas palestinien comme ailleurs, n'implique pas qu'aucun processus démocratique partiel ne puisse exister.

4 En faisant la sociologie des candidatures présentées lors de ces élections en une dizaine d'années et l'analyse des scores obtenus par les diverses formations politiques, je chercherai à voir si les spécificités d'un tel échantillon demeurent irréductibles à la scène politique globale ou, au contraire, dessinent des équilibres longtemps limités aux seuls campus et soudainement étendus à l'ensemble de la société par le soulèvement des trois dernières années.

Structures des conseils étudiants, modes de scrutin et électionsLes structures et les modes de scrutin

5 Cinq grandes universités regroupent la quasi-totalité des étudiants du supérieur en Cisjordanie, soit près de 10000 hommes et femmes pour l'année qui précède le soulèvement ${ }^{4}$ : université nationale Najâh de Naplouse, universités de Bir Zeit et de Bethléem, université islamique d'Hébron et Institut Polytechnique d'Hébron ${ }^{5}$. Elles ont toutes un conseil étudiant élu, généralement constitué de 9 membres, à l'exception de celui de Najâh qui en compte $11^{6}$. Les élections ont lieu normalement en début d'année universitaire, sauf à Bethléem où les étudiants élisent leur conseil au mois de mai pour l'année universitaire qui suit Le rythme de ces élections a cependant été brisé, dans la majorité des cas, à cause des multiples fermetures des universités par ordre militaire.

6 Les modes de scrutin diffèrent selon les universités. A Najâh, lors de sa création en 1978, le conseil comptait 9 membres non élus, désignés pour représenter chaque groupe politique connu. Une réforme adoptée en 1979 a fait passer de 9 à 11 le nombre de membres du conseil : un président, un vice-président, un secrétaire, un chargé des relations publiques ainsi que les responsables des divers comités (finances, affaires sociales, sport, restaurant et cafétéria, sécurité, culture et art). Cette réforme a également institué le mode de scrutin : l'élection se fait à la majorité relative sur des listes non bloquées. Najâh possède également une assemblée générale, constituée de 56 
membres élus dans chaque département, qui a capacité de débattre et d'aviser le conseil ; elle approuve le budget de ce dernier et peut le démettre par un vote des deux tiers?.

7 A Bir Zeit, le conseil, créé en 1971, compte 9 membres élus depuis une réforme adoptée en 1979: un secrétaire général et 8 secrétaires de comités (culture, art, finances et bourses, cafétéria, travail volontaire, affaires sociales, affaires académiques et relations publiques). Tous sont élus par l'ensemble des étudiants inscrits au scrutin majoritaire à un tour sur des listes bloquées.

8 A Bethléem, le conseil, créé en 1975, se compose de 4 membres "administratifs" (président, vice-président, trésorier et secrétaire), élus individuellement à la majorité relative par l'ensemble des étudiants inscrits. Les 5 autres membres du conseil sont les secrétaires des «clubs» (culture, sports, folklore palestinien, art et affaires sociales); tous sont élus à titre individuel et à la majorité relative par les seuls étudiants inscrits dans chacun des clubs ; un trésorier et un membre du bureau sont également élus dans chaque club pour accompagner le secrétaire dans sa gestion sans avoir, cependant, le droit de participer au conseil plénier.

9 A I'université islamique d'Hébron, les élections se font au scrutin majoritaire à un tour sur des listes non bloquées. Le conseil, créé en 1975 mais dont les premières véritables élections se tiennent en 1982, comprend 9 membres : un président, un vice-président, un secrétaire, un trésorier et les présidents des comités : culture, affaires sociales, sport et voyages, entraide et affaires des étudiants.

10 Le conseil étudiant de l'Institut polytechnique est également élu au scrutin majoritaire à un tour sur des listes non bloquées et comprend 9 membres : un président, un viceprésident, un secrétaire, un trésorier, un chargé des activités universitaires ainsi que les présidents des comités culture, art, affaires sociales et sport.

La constitution des listes

11 L'ensemble des grandes forces organisées de l'échiquier politique palestinien de l'Extérieur se retrouvent en compétition dans les élections universitaires de l'Intérieur, cachées sous des étiquettes qui, en réalité, ne trompent personne: Harakat al-Chabiba al-Tullâbiyya (Mouvement étudiant de la jeunesse) défend les positions du Fath ; Jabhat al-Ittifhâd wa-l-Taqaddum (Front de l'union et du progrès) celles du Parti communiste palestinien; Jabhat al-'Amal al-Tullâbî (Front d'action étudiante) celles du Front Populaire de Libération de la Palestine (FPLP de Georges Habache) ; Kutlat al-Wahda alTullâbiyya (Bloc de l'unité étudiante) celles du Front Démocratique de Libération de la Palestine (FDLP de Nayef Hawatmeh) ; al-KutIa al-lslâmiyya (Bloc islamique) celles des Frères musulmans. Les partisans du Fath-Commandement révolutionnaire d'Abû Mûsâ n'ont présenté de listes indépendantes qu'à 3 occasions, en janvier 1985 à Bir Zeit, en décembre 1985 à Hébron et en juillet 1986 à Najâh; certains membres de ces listes avaient déjà participé à des élections et été élus sous étiquette Fath. A Bethléem, les listes du FPLP ont souvent été des listes "du refus ", incluant aux côtés des partisans de Georges Habache des militants du Front Populaire de Libération de la PalestineCommandement Général (FPLP-CG d'Ahmad Jibril), du Front de Libération Populaire de la Palestine (FLPP de Samir Ghoché), du Parti communiste révolutionnaire ou encore de la Saïqa. A Bethléem et à l'université islamique d'Hébron, des candidats "indépendants» ont également eu l'occasion de participer à des scrutins; bien que refusant de revendiquer une quelconque affiliation à une organisation membre de 
l'OLP, ils sont, cependant, toujours connus comme proches de telle ou telle tendance nationaliste. A aucun moment des candidats n'ont défendu les positions de la Jordanie.

Dans les 5 établissements, l'ensemble des blocs en lice ont la possibilité de s'entendre avant le scrutin et de proposer une liste unitaire. Les élections n'ont alors pas lieu, les candidats étant considérés comme élus (mode de tazkiyya). Les blocs peuvent également négocier des listes d'union partielle ou d'«union nationale», les islamistes ayant toujours fait cavalier seul.

13 Les listes, surtout lorsqu'elles sont d'union, portent un nom différent à chaque élection qui fait référence presque toujours à un événement marquant de l'année politique : «liste des martyrs du Beaufort» (position de l'OLP au Sud-Liban, prise lors de l'invasion de 1982), «liste Fahd al-Qawasmeh» (maire banni d'Hébron assassiné à Ammân), «liste Kanafânî » (haut responsable du FPLP assassiné à Beyrouth), etc., ou simplement: "liste de l'olivier», chacun retrouvant immédiatement les affiliations politiques implicites. Dans tous les autres cas, la liste porte le nom du bloc dont elle défend les positions.

14 Seul le nom du bloc dont se réclame chacune des listes peut donner une indication précise de l'affiliation politique; dans la majorité des cas, en effet, les programmes publiés par les listes sont exclusivement consacrés à des questions touchant à la seule vie universitaire et aux problèmes quotidiens de l'étudiant (ouverture de cafétéria, de services de photocopies, amélioration des transports en commun, etc.) et quasiment rien ne les distingue entre eux; le débat politique qui constitue le véritable enjeu de l'élection a lieu lors des meetings organisés sur les campus.

Sources de l'étude

Ce travail repose sur le dépouillement systématique des résultats publiés dans la presse palestinienne qui n'a, cependant, reproduit que très rarement les données complètes des scrutins; assez souvent, les informations publiées sont contradictoires, pas seulement entre journaux mais parfois à l'intérieur d'un même périodique. Je me suis référé à l'ensemble de la presse de l'Intérieur : les trois grands quotidiens, al-Quds, alFajr et al-Cha'b (al-Anbâ', quotidien publié en arabe par le gouvernement israélien et maintenant disparu, ne publiait pas ce genre d'informations) ainsi qu'aux hebdomadaires, al-'Awda, al-Bayâdir al-Siyâsî et al-Talî́a (en arabe) ainsi qu'al-Fajr weekly (en anglais). J'ai également utilisé, au coup par coup, les publications des organisations palestiniennes de "l'Extérieur " (Democratic Palestine, tout particulièrement) ainsi que la presse jordanienne, al-Râyy et al-Dustûr. La presse israélienne n'a jamais rendu compte de ces élections dans le détail. Les universités, quant à elles, n'ont que très rarement publié les résultats dans leur bulletin d'information; lorsqu'elles l'ont fait, cependant, c'est toujours leurs chiffres et leurs noms qui ont été retenus comme critère de véracité des informations parues dans la presse.

Si le nom de la liste sur laquelle les candidats ont été élus figure presque toujours, l'appartenance politique individuelle du candidat en cas de liste d'union n'apparaît jamais, pas plus que sa résidence, sa confession ni sa faculté d'origine, critères que j'ai retenus comme discriminants. J'ai donc complété mon information par des interviews d'anciens élus et de membres de l'administration universitaire ${ }^{8}$. L'inexistence d'archives propres aux conseils (due soit aux confiscations opérées par l'armée lors de descentes, soit à la négligence) ou l'impossibilité $d$ 'y avoir accès, de même que l'inaccessibilité des fichiers tenus par les administrations des universités excluent, 
malheureusement, la fiabilité absolue des renseignements obtenus par interviews et empêchent l'exhaustivité des données.

Sociologie des candidaturesétat général de l'échantillon

17 Le tableau 1 livre un état général de l'échantillon ici réuni (voir tableaux en annexe. NB : l'intégralité des tableaux détaillés sur lesquels s'appuie cette étude n'a pu être reproduite ici. Ils figurent dans les Actes du colloque mentionné plus haut, à paraître dans les dossiers du CEDEJ). Les périodes chronologiques couvertes varient selon les établissements considérés pour des raisons touchant aux sources disponibles. En ce qui concerne Najâh et Hébron, l'étude couvre l'ensemble des élections tenues de même que pour Bir Zeit (si l'on commence avec la réforme du mode de scrutin); en revanche, l'absence de sources disponibles antérieures à 1978 pour Bethléem (le conseil ayant été fondé en 1975) et à 1981 pour Polytechnique (dont le conseil fonctionne au moins depuis 1980) empêche une même exhaustivité chronologique.

18 A cause de l'impression des sources, les chiffres donnés dans les colonnes "Fath", «FPLP », «FDLP » et «PCP » des tableaux peuvent être inférieurs à la réalité, la différence se retrouvant partiellement avec les colonnes "gauche " (FPLP. plus FDLP, plus PCP, plus Abu Mûsâ et les divers groupes du Front du refus, plus les "indépendants» de Bethléem, plus les militants de gauche sans autre précision) et «Nationalistes» (Fath, plus gauche, plus indépendants, plus les militants non islamistes sans autre précision). Les colonnes «nationalistes » et «islamistes » sont dans la quasi-totalité des cas conformes à la réalité (12 « non-identifiés » politiquement demeurent en bout de traitement et constituent la différence entre le "total » et l'addition des « nationalistes » et des « islamistes »).

Dans les 5 universités concernées, j'ai identifié la totalité des candidatures élues, soit 365 cas. J'ai ensuite tenté d'élargir au maximum cet échantillon, exhaustif mais limité en importance numérique, en retrouvant dans la mesure du possible l'identité des candidats non élus. Si l'on met à part l'université de Bethléem, la proportion de cas identifiés par rapport à l'ensemble des candidatures présentées est de $76 \%$ mais varie selon les établissements : si elle atteint plus de $91 \%$ à Bir Zeit, elle n'est que de $68 \%$ à Najâh et à Hébron et de 55\% à Polytechnique. L'échantillon retenu à Bethléem constitue un cas particulier puisqu'il est constitué de la totalité des candidatures élues, non seulement celles des membres du conseil plénier mais également des élus au sein des clubs dépourvus du droit de participation au conseil ; le nombre de candidatures non élues étudié y est en revanche quasiment nul. Des inégalités dans l'identification se retrouvent également selon les appartenances politiques. A Najâh, par exemple, nombreux sont les noms de candidats non élus de gauche ou islamistes effacés des mémoires ; à Bethléem, c'est l'identité des candidats du Fath qui fait surtout défaut.

Ces limites de l'échantillon interdisent donc toute lecture qui considérerait les chiffres en soi ; les tableaux ne peuvent parler que par la mise en rapport des chiffres obtenus : seules les disproportions ou les adéquations peuvent être prises en compte, non pas les chiffres en eux-mêmes. Ces limites connues, les tendances manifestées par l'échantillon élargi ne perdent pas pour autant toute représentativité : aucune véritable rupture de tendance, en effet, n'apparaît entre les deux échantillons, élus d'une part et non élus de l'autre, dans l'ensemble des domaines considérés'. On peut donc légitimement considérer que les tendances manifestées par l'échantillon exhaustif des 365 candidatures élues se retrouvant de manière quasi identique dans l'échantillon élargi des 720 candidatures, ces mêmes tendances se retrouveraient dans cet autre 
échantillon exhaustif, mais impossible à établir dans l'état des sources, constitué de l'ensemble des candidatures élues et non élues présentées durant la période considérée.

21 Cette étude porte sur les candidatures et non sur les candidats. Le taux de candidatures multiples présentées par un même candidat oscille selon les universités entre $15 \%$ et $6 \%$ (si l'on excepte Bir Zeit où le taux dépasse très largement la moyenne du fait que presque la moitié des candidats islamistes y ont présenté des candidatures successives). 624 des 720 candidatures étudiées ont été des candidatures uniques; 78 candidats se sont présentés deux fois, 17 trois fois et 1 quatre fois. De façon générale, les islamistes présentent plus de candidatures multiples que les nationalistes, de même qu'un candidat malheureux à une première élection se représente une seconde fois plus souvent qu'un élu du premier coup (ce trait pourrait rendre compte du nombre important de candidatures multiples chez les islamistes, globalement élus moins souvent).

Le grand nombre de candidatures uniques indique un renouvellement important des cercles politiquement actifs sur les campus; ce roulement accéléré des candidatures peut être dû à l'importance du nombre d'étudiants désireux d'un engagement politique universitaire et à la nécessité de leur offrir une place de responsabilité, et/ou à la prise en considération des risques encourus par un étudiant trop longtemps demeuré au premier rang de l'activisme estudiantin. L'étude de la répression israélienne à l'encontre des milieux étudiants confirme cette hypothèse ${ }^{10}$. Au moins 96 des 365 candidatures élues (soit $25 \%$ de l'échantillon, chiffre sans aucun doute bien en-deçà de la réalité) ont en effet été victimes de la répression israélienne sous ses différentes formes (condamnation à des peines d'emprisonnement ou à des amendes, détention administrative, assignation à domicile, bannissement); les mêmes recherches appliquées) à l'échantillon de l'ensemble des candidatures donnant environ un taux de victimes de la répression de l'ordre de $18 \%$ (137 des 720 candidatures), on peut en conclure que le fait d'être élu augmente les risques de répression ${ }^{11}$.

Le sexe

23 Dans tous les cas, avec seulement $11 \%$, le pourcentage de candidatures féminines est très largement inférieur à celui des inscriptions, les femmes constituant près de $40 \%$ des effectifs universitaires ${ }^{12}$. La disproportion réelle est encore plus importante puisque, contrairement aux facultés scientifiques et religieuses dont les effectifs sont massivement masculins, les facultés des Lettres et les facultés d'Economie qui fournissent les contingents les plus importants de candidatures sont à majorité féminine.

24 La sous-représentation des femmes, quoique chronique, varie selon les établissements. A Najâh, en l'état de la documentation, seules 3 étudiantes ont, en 9 ans, posé leur candidature (aux côtés de 170 hommes). A Polytechnique, 2 étudiantes seulement se sont portées candidates (aux côtés de 61 hommes); dans ce cas d'espèce, cependant, la disproportion peut être rapportée au caractère massivement masculin de cette université d'ingénierie qui ne compte que $5 \%$ de femmes ${ }^{13}$. Si Hébron laisse une place un peu moins réduite à ses étudiantes ( 6 sur 80 candidatures), c'est Bir Zeit, aux effectifs masculins globalement plus importants que les autres universités (à l'exception de Polytechnique) et Bethléem, surtout, qui atténuent la distorsion: le déficit de participation politique féminine par rapport à l'ensemble des effectifs universitaires atteint à Najâh $40 \%$ et à Hébron $33 \%$; il n'est plus que de $24 \%$ à Bir Zeit et de $21 \%$ à Bethléem. 
25 Les variations du degré de participation politique féminine entre établissements peuvent être rapportées à celles des taux de la présence globale des chrétiens et des résidents de la conurbation de Jérusalem dans chacune de ces universités : les femmes sont beaucoup plus engagées politiquement à Bethléem et à Bir Zeit qu'à Najâh et à Hébron.

26 La confession, sans aucun doute, entre en ligne de compte pour une part importante dans l'engagement des femmes (tableau 2) puisque les chrétiens sont presque cinq fois plus nombreux chez les femmes que chez les hommes: les chrétiens, en effet, ne constituent que $9 \%$ de l'échantillon masculin élargi alors qu'ils constituent $42 \%$ de l'échantillon féminin (respectivement $8 \%$ et $32 \%$ de l'échantillon des seuls élus); de même, $36 \%$ de l'échantillon chrétien élargi sont des femmes, étudiantes qui ne constituent que $7 \%$ de l'échantillon musulman (respectivement $27 \%$ et $7 \%$ de l'échantillon des seuls élus).

27 La résidence dans le périmètre Ramallâh, Jérusalem, Bethléem (tableau 4) constitue également un élément important dans le degré d'engagement politique estudiantin des femmes puisque $71 \%$ d'entre elles habitent cette région contre seulement $32 \%$ des hommes (respectivement $59 \%$ et $27 \%$ des élus). La citadinité, en tant qu'élément superposé au lieu de résidence limité au centre de la Cisjordanie, apparaît elle aussi liée au pourcentage de participation féminine : $77 \%$ des femmes candidates ( $63 \%$ des élues) résident en ville contre seulement $46 \%$ des hommes (41\% des élus) (tableau 3); inversement, seules $13 \%$ des candidates sont rurales contre $45 \%$ des hommes (respectivement $17 \%$ et $49 \%$ des élus).

28 La résidence dans un camp de réfugiés semble également jouer un rôle important dans l'engagement féminin, indépendamment de la caractéristique confessionnelle puisque $2 \%$ seulement des chrétiens candidats résident dans les camps : les femmes constituent $13 \%$ de l'échantillon élargi des résidents des camps (17\% des élus); $10 \%$ de l'échantillon féminin élargi ( $20 \%$ des élues) résident ainsi dans les camps.

Ces trois traits qui caractérisent l'échantillon féminin peuvent également être mis à contribution pour rendre compte des variations de l'engagement féminin selon les établissements. Du point de vue de la confession, les chrétiens, hommes et femmes, sont quasiment absents de Najâh, d'Hébron et de Polytechnique, universités dans lesquelles la participation des femmes connaît son minimum ; à Bir Zeit, en revanche, les chrétiens sont présents ( $6 \%$ de l'échantillon élargi, $10 \%$ des élus) et surtout à Bethléem (35\% de l'échantillon élargi, 32\% des élus), deux universités qui limitent la sous-représentation féminine.

30 Le lieu de résidence intervient également: Bir Zeit, par exemple, compte $31 \%$ de candidatures dont les titulaires résident dans la conurbation de Jérusalem (36\% des élus); à Bethléem, le phénomène est encore plus fort puisque $80 \%$ des candidatures sont présentées par des résidents de la région de Jérusalem (79\% des membres élus au conseil plénier). A l'inverse, les universités qui font peu de place aux femmes ne comptent que relativement peu de candidatures présentées par des résidents de la région de Jérusalem : $2 \%$ à Najâh (et aucun élu), 10\% à Hébron (et autant d'élus), $14 \%$ à Polytechnique (et $12 \%$ d'élus).

31 Le degré de citadinité, enfin, couplé à une résidence dans les régions Centre de la Cisjordanie, ne fait que corroborer cette remarque: on s'aperçoit en effet que les candidatures à Najâh, à Hébron et à Polytechnique sont à 54\%-57\% le (ait de ruraux et à 
33\%-39\% seulement de citadins. A Bethléem, à l'inverse, $67 \%$ des candidatures ont été présentées par des citadins pour seulement $22 \%$ de candidatures présentées par les ruraux. A Bir Zeit, le type de résidence ne semble jouer que dans une moindre mesure avec $47 \%$ de candidatures rurales pour seulement $44 \%$ de candidatures citadines. La citadinité couplée à une résidence dans les régions Nord et Sud, en revanche, ne semble pas déboucher, de façon générale, sur l'engagement politique féminin, les islamistes faisant exception puisque leurs 4 uniques candidates résident dans les villes du Nord et du Sud. Le fait d'être chrétien l'emporterait ainsi sur la citadinité dans l'ordre d'importance des causes de l'engagement féminin.

Politiquement, si l'on considère l'échantillon élargi où les femmes constituent $11 \%$ des candidatures (9\% des élus), ce sont les listes islamistes qui comptent le moins d'étudiantes avec seulement 3\% de candidatures féminines (et également 3\% des élus) (tableau 2). Aucun candidat islamiste, bien évidemment, n'est chrétien, et $12 \%$ seulement d'entre eux résident dans les régions Centre, caractéristiques qui, a contrario, confirment l'hypothèse avancée plus haut sur le rôle de la confession et de la résidence dans l'engagement féminin; 3 des 4 femmes identifiées engagées sur des listes islamistes sont citadines, ce qui confirmerait l'importance de la citadinité dans l'engagement politique féminin dans le cadre de mouvements religieux conservateurs, élément cependant limité par la pression confessionnelle.

Le Fath se situe également au-dessous de la moyenne de participation féminine avec seulement $9 \%$ de candidatures présentées par des femmes (7\% des élus): Cette caractéristique peut être mise en relation avec une participation généralement faible des chrétiens à cette organisation alors même que 8 des 20 candidates Fath sont de confession chrétienne (soit $40 \%$ de l'échantillon féminin du Fath face à seulement $4 \%$ de son échantillon masculin et à $13 \%$ de l'échantillon des deux sexes). Elle peut être également rapprochée du petit nombre de candidatures présentées par des résidents de la conurbation de Jérusalem au titre du mouvement, alors même que 13 des 20 candidatures Fath présentées par des femmes l'ont été par des résidentes de cette zone (soit $65 \%$ de l'échantillon féminin Fath pour seulement 17\% de son échantillon masculin et $36 \%$ de l'échantillon des deux sexes et de toutes tendances). Le très fort taux de ruraux parmi les échantillons du Fath, enfin ( $58 \%$ face à $41 \%$ pour l'échantillon toutes tendances, $61 \%$ face à $46 \%$ des élus) et la faible présence citadine (32\% face à $50 \%, 27 \%$ face à $52 \%$ des élus), peuvent également rendre compte de cette faible participation féminine; l'échantillon féminin du Fath, d'ailleurs, inverse les proportions observées dans l'échantillon masculin : 49 citadins pour 107 ruraux chez les hommes (soit $29 \%$ et 63\%), 12 citadines pour seulement 3 rurales chez les femmes (soit $60 \%$ et $25 \%$ ). La participation de femmes résidant dans les camps dans les rangs du Fath doit également être ici soulignée : les réfugiés, en effet, constituent $25 \%$ de l'échantillon féminin élargi du Fath pour seulement 9\% de l'échantillon masculin et $9 \%$ de l'échantillon toutes tendances (respectivement 33\%, 10\% et $12 \%$ de l'échantillon des élus).

Le FDLP se situe légèrement au-dessus de la moyenne de la participation féminine avec $13 \%$ de femmes dans l'échantillon élargi (et exactement dans la moyenne de l'échantillon des élus avec 9\%). L'élément confessionnel joue un rôle important : 3 des 8 candidatures présentées par des femmes au nom du FDLP sont chrétiennes et l'une de ses 2 élues (soit $38 \%$ et $50 \%$ de l'échantillon féminin FDLP contre $9 \%$ et $20 \%$ de son échantillon masculin et $13 \%$ et $10 \%$ de l'échantillon des deux sexes toutes tendances); au niveau de l'échantillon élargi, hommes et femmes, $13 \%$ des candidatures présentées 
par le FDLP l'ont été par des chrétiens qui constituent 23\% des élus du bloc (face à $13 \%$ et $10 \%$ de moyenne). La résidence dans la conurbation de Jérusalem joue également un grand rôle : 59\% des candidatures du FDLP ont été présentées par des résidents de la région de Jérusalem (pour $36 \%$ de moyenne générale) et 5 de ses 7 candidates y habitent (soit $71 \%$ de l'échantillon féminin contre $58 \%$ de l'échantillon masculin). Comme ailleurs, la citadinité joue son rôle puisque 5 des 6 candidatures féminines identifiées l'ont été par des citadines (soit $83 \%$ de l'échantillon féminin contre $53 \%$ de l'échantillon masculin).

Avec 19\% de l'échantillon élargi et $11 \%$ de l'échantillon des élus, le PCP donne aux femmes une place très nettement supérieure à la moyenne. Une fois de plus, la confession joue sans aucun doute un rôle important dans cette mobilisation des femmes: 5 des 10 candidatures féminines présentées par le PCP l'ont été par des chrétiennes (contre $20 \%$ de l'échantillon masculin). Les chrétiens constituent $26 \%$ de l'échantillon élargi du PCP et $30 \%$ de ses élus, la moyenne générale se situant respectivement à $13 \%$ et $10 \%$. La résidence dans la conurbation de Jérusalem apparaît là encore comme caractéristique de l'engagement féminin: au moins 6 des 10 candidatures féminines du PCP ont été présentées par des résidentes de cette zone (soit $66 \%$ de l'échantillon féminin du PCP face à seulement $49 \%$ de l'échantillon masculin et contre 36\% de l'échantillon des deux sexes toutes tendances). La citadinité caractérise également les femmes qui ont posé leurs candidatures pour le compte du PCP : 6 des 9 candidatures ont été présentées par des citadines (soit $67 \%$ de l'échantillon féminin du PCP face à $62 \%$ de son échantillon masculin et à $50 \%$ de l'échantillon des deux sexes toutes tendances).

La participation féminine est de très loin la plus forte dans les rangs du FPLP avec $21 \%$ de ses deux échantillons. Le fort taux de chrétiens dans ses rangs peut sans doute rendre compte de ce très haut niveau de participation féminine : au moins 16 des 29 candidatures féminines du groupe ont été présentées par des chrétiennes (soit 57\% de l'échantillon féminin du FPLP face à $24 \%$ de son échantillon masculin et à $13 \%$ de l'échantillon des deux sexes toutes tendances). De même, le très grand nombre de résidents de la conurbation de Jérusalem explique-t-il cette participation des femmes : au moins 25 des 28 candidatures féminines identifiées du FPLP l'ont été par des étudiantes qui habitent cette région (soit $90 \%$ de l'échantillon féminin du FPLP face à $62 \%$ de son échantillon masculin et à $36 \%$ seulement de l'échantillon des deux sexes toutes tendances). La citadinité ne fait, une fois encore, que renforcer le phénomène : 25 des 27 candidatures féminines du FPLP ont été présentées par des citadines (soit 93\% de l'échantillon féminin du FPLP face à $59 \%$ de son échantillon masculin et à $47 \%$ de l'échantillon des deux sexes toutes tendances).

La confession

37 Le tableau 2 montre une participation de candidats de confession chrétienne de l'ordre de $13 \%$ de l'échantillon élargi et de $10 \%$ de l'échantillon des seuls élus. Si l'on rapporte ces chiffres à ceux de la population de Cisjordanie parmi laquelle les chrétiens ne dépassent pas les $5 \%{ }^{14}$, apparaît une surreprésentation chrétienne due essentiellement à l'université de Bethléem (où ont été présentées 79 des 91 candidatures de chrétiens, et 29 des 37 candidatures élues). Malgré l'absence de statistiques confessionnelles concernant la population universitaire, il semble fondé d'affirmer que cette surreprésentation est un phénomène global à l'Université, qui apparemment recrute proportionnellement beaucoup de chrétiens, mais ne constitue pas une distorsion dans 
la représentation de la seule population universitaire. Selon Mûsâ Darwîch, porteparole de l'université de Bethléem ${ }^{15}$, son établissement compterait environ $35 \%$ de chrétiens, taux qui correspond exactement à celui des candidatures qui y ont été présentées par des chrétiens; ainsi, malgré des conditions extrêmement favorables propres à cette université catholique gérée par les Frères des Ecoles chrétiennes et le Vatican, aucune surreprésentation chrétienne ne peut être observée à Bethléem, les chrétiens ne constituant même que 32\% des élus. A Bir Zeit, où seulement 10 des 158 candidatures étudiées ont été le fait de chrétiens (soit 6\% de l'effectif élargi et 10\% des élus), c'est sans aucun doute d'une sous-représentation qu'il s'agit, l'université, propriété d'une riche famille chrétienne de ce village situé à proximité de Ramallâh et de Jérusalem à fort taux de population chrétienne, comptant une importante proportion de chrétiens. Ce phénomène peut s'expliquer par la géographie politique propre à cette université : face à un élément islamiste très actif, des listes d'union ont souvent vu le jour sous égide du Fath, organisation très peu marquée par l'élément chrétien. La quasi absence de chrétiens à Najâh et à l'université islamique d'Hébron ainsi que leur absence totale à Polytechnique est «normale » du fait de la présence quasi négligeable de communautés chrétiennes dans le nord et le sud de la Cisjordanie d'où proviennent la majorité des effectifs de ces universités.

La caractéristique première de ces candidatures présentées par des chrétiens est la résidence massivement regroupée dans la conurbation de Jérusalem : $94 \%$ des candidats chrétiens y habitent (contre seulement $26 \%$ des musulmans de l'échantillon élargi, $88 \%$ contre $24 \%$ de l'échantillon des seuls élus). La répartition selon le type de résidence découle de cette caractéristique : $90 \%$ des chrétiens sont citadins contre seulement $42 \%$ des musulmans de l'échantillon élargi (81\% contre 38\% de l'échantillon des seuls élus), les chrétiens constituant ainsi $29 \%$ de l'échantillon élargi des citadins pour $13 \%$ seulement de l'échantillon global ( $23 \%$ contre $10 \%$ de l'échantillon des seuls élus). $8 \%$ des chrétiens seulement sont ruraux contre $47 \%$ des musulmans ( $16 \%$ et $50 \%$ de l'échantillon des seuls élus) et $2 \%$ habitent les camps contre $10 \%$ des musulmans ( $3 \%$ contre $13 \%$ de l'échantillon des seuls élus).

Comme souligné précédemment, les candidatures présentées par des chrétiens peuvent être caractérisées par un taux de participation féminine beaucoup plus important que dans le cas de l'échantillon musulman : $42 \%$ des femmes engagées politiquement sont, en effet, de confession chrétienne pour $9 \%$ seulement des hommes (32\% contre $8 \%$ de l'échantillon des seuls élus), les femmes constituant ainsi $36 \%$ de l'échantillon chrétien élargi contre $7 \%$ seulement de l'échantillon musulman ( $27 \%$ et $7 \%$ de l'échantillon des seuls élus).

De façon générale, les chrétiens présentent $17 \%$ des candidatures «nationalistes » et $25 \%$ des candidatures de la "gauche » (respectivement $13 \%$ et $27 \%$ des élus). $47 \%$ des chrétiens se sont présentés sous l'égide du FPLP dont ils assurent $31 \%$ des candidatures (24\% de l'échantillon des élus). Le PCP se situe en seconde position avec $26 \%$ de candidatures présentées par des chrétiens ( $30 \%$ de l'échantillon des élus). Le FDLP, malgré l'appartenance confessionnelle chrétienne de Nayef Hawatmeh, son secrétaire général, ne sur-représente pas les chrétiens qui constituent $13 \%$ de ses candidatures (et $18 \%$ de ses élus). Le Fath, enfin, peut être caractérisé par une très faible participation chrétienne avec seulement $7 \%$ de son échantillon élargi et $4 \%$ de ses élus.

Le lieu de résidence 
41 La géographie du lieu de résidence de l'ensemble de la population étudiante varie considérablement selon les universités. Najâh manifeste un recrutement limité quasi exclusivement aux régions Nord de la Cisjordanie avec $85 \%$ de ses effectifs résidant dans les régions de Naplouse, Jénine et Tûlkarm, auquel se joint un contingent relativement important d'étudiants venus de la Bande de Gaza (8\%). Une réputation de qualité moyenne, l'éloignement géographique, et la présence des universités de Bir Zeit et de Bethléem font que peu d'étudiants résidents des régions Centre et Sud font le déplacement de Naplouse. Constituant 52\% de l'échantillon élargi (et 55\% des élus), les étudiants habitant Naplouse ainsi que ceux de la Bande de Gaza (11\% et $9 \%$ ) font preuve d'un engagement politique proportionnellement plus important que leur présence dans les effectifs globaux à l'inverse de leurs collègues de Tûlkarm et de Jénine (qui adoptent une conduite contraire à Bir Zeit).

Bir Zeit tait preuve de la plus grande diversité géographique dans le recrutement de ses effectifs. L'antériorité de sa fondation sur les autres universités, sa réputation de sérieux dans l'enseignement, son image hautement positive dans la conscience nationaliste et l'absence de connotation confessionnelle marquée malgré la confession de ses propriétaires, constituent sans aucun doute des facteurs qui expliquent cette ouverture. Les résidents de la Bande de Gaza y sont majoritairement inscrits dans les facultés scientifiques, absentes de l'organigramme de l'université islamique de Gaza. Tandis que les candidats résidents des régions Nord manifestent un engagement politique plus important que leur part dans les effectifs globaux et connaissent une surreprésentation encore plus forte parmi les seuls élus, ceux des régions Sud présentent un sous-engagement politique évident; les étudiants venus de la Bande de Gaza, quant à eux connaissent une surreprésentation dans l'échantillon élargi mais une forte sous-représentation dans l'échantillon des seuls élus due à leur engagement majoritaire parmi les islamistes toujours exclus des conseils. Les étudiants de Jénine et de Tûlkarm, à l'inverse de leur attitude à Najâh, manifestent également un engagement politique important.

43 L'université de Bethléem, un peu à l'image de Najâh, recrute la majorité de ses effectifs dans sa propre région, $84 \%$ de ses effectifs résidant entre Jérusalem au nord et Hébron au sud, élément qui recoupe la géographie confessionnelle de la Cisjordanie. L'identité confessionnelle fortement marquée de l'administration, du corps enseignant (de nombreux Frères et jésuites) et des étudiants ( $35 \%$ des effectifs comme souligné plus haut) font sans doute préférer les autres universités à des milieux musulmans traditionalistes tandis que la population chrétienne groupée dans les régions Centre n'hésite pas à mettre ses jeunes entre les mains du clergé. Les islamistes, d'ailleurs, ne sont parvenus que récemment à prendre le contrôle, à deux reprises, du petit club sportif (voir infra). Les étudiants résidant dans la région de Bethléem apparaissent légèrement plus engagés politiquement (ainsi que ceux de Ramallâh et de Jénine) que leurs collègues des autres régions.

La bi-polarité géographique des effectifs de l'université islamique d'Hébron, avec ses $56 \%$ d'étudiants résidents de la région Sud et ses $27 \%$ des régions Nord, s'explique sans doute par son caractère confessionnel marqué qui attire les milieux musulmans traditionnels principalement répandus dans ces deux zones qui ne connaissent ni sousreprésentation ni surreprésentation évidente.

Polytechnique, enfin, connaît également une bipolarité Nord-Sud, tempérée cependant par la présence d'un fort contingent venu de la Bande de Gaza dépourvue de faculté 
scientifique. Les régions Centre connaissent une large sous-représentation dans les effectifs globaux, accentuée ensuite au niveau des échantillons tant élargi que restreint aux élus. A l'inverse, les étudiants de la Bande de Gaza comme ceux de Jénine y manifestent un engagement politique largement supérieur à leur taux de présence parmi les effectifs globaux.

Si .l'on met en relation, d'une part, l'importance de l'échantillon des résidents d'un llwâ donné au sein de l'échantillon élargi, toutes tendances politiques confondues, et son importance au sein de l'échantillon d'une tendance politique précise, et d'autre part, l'importance de l'échantillon d'une tendance politique précise au sein de l'échantillon élargi, toutes résidences confondues, et son importance au sein de l'échantillon des résidents d'un llwâ donné, les tendances générales de chaque llwâ apparaissent d'autant plus clairement que sont fortes les distorsions. Le tableau 4 fait apparaître de tels déséquilibres ${ }^{16}$. Les limites déjà soulignées des échantillons utilisés empêchent bien évidemment de dresser une carte figée de l'échiquier politique pour chacun des llwâ de Cisjordanie; de solides tendances peuvent cependant se dessiner pour chacun d'eux. Il convient, malgré tout, de mettre à part le cas de l'échantillon des résidents de Gaza : seuls les étudiants de facultés scientifiques sont contraints de faire le voyage de Cisjordanie, facultés connues par ailleurs, et indépendamment du lieu de résidence, comme fortement impliquées dans le mouvement islamiste.

Le llwâ de Tûlkarm, à l'évidence, sur représente le Fath : 52\% des résidents de Tulkarm ont défendu les positions du Fath, lequel ne constitue que 33\% de l'échantillon toutes résidences ; le llwâ qui a fourni $9 \%$ de l'échantillon toutes tendances constitue ainsi $14 \%$ de l'échantillon Fath. Le contingent d'islamistes est également plus important que la normale, mais dans une moindre mesure: alors que les Frères musulmans ne constituent que $23 \%$ de l'échantillon toutes résidences, ils fournissent $28 \%$ de l'échantillon Tûlkarm; parallèlement, alors que, toutes tendances politiques confondues, les résidents de Tûlkarm constituent $9 \%$ de l'échantillon élargi, le taux monte à $11 \%$ pour le seul échantillon islamiste. La gauche, globalement, paraît fortement sous-représentée : $17 \%$ seulement des résidents de Tûlkarm ont défendu les positions des FPLP, FDLP et PCP, lesquels constituent $42 \%$ de l'échantillon toutes résidences; ce déficit est presque entièrement imputable à la faiblesse du FPLP qui, bien que constituant $22 \%$ de l'échantillon toutes résidences, n'a patronné que $3 \%$ des candidatures de Tûlkarm.

Le llwâ de Jénine accorde également une forte surreprésentation au Fath, relativement moins importante cependant que celle observée à Tûlkarm: $43 \%$ des candidatures présentées par ses résidents ont soutenu le Fath qui ne constitue que $33 \%$ de l'échantillon toutes résidences; $11 \%$ de l'échantillon Fath sont ainsi des résidents de Jénine, lesquels ne constituent que $9 \%$ de l'échantillon toutes tendances. Grâce au FPLP et dans une moindre mesure au FDLP, mais malgré le PCP, la gauche tient une place légèrement plus importante que la normale à Jénine : $43 \%$ des candidats de la région l'ont soutenue, elle qui constitue $42 \%$ de l'échantillon élargi. A l'inverse, les islamistes y apparaissent comme sous-représentés : $11 \%$ seulement des résidents de Jénine ont défendu les idées des islamistes qui constituent $23 \%$ de l'échantillon toutes résidences ; les résidents de Jénine qui constituent $8 \%$ de l'échantillon toutes tendances, ne fournissent ainsi que $4 \%$ de l'échantillon islamiste.

Le llwâ de Naplouse accorde au Fath une surreprésentation du même ordre que celle observée chez les résidents de la région de Jénine : $44 \%$ de ses candidatures ont soutenu 
le Fath, lequel ne constitue que $33 \%$ de l'échantillon toutes résidences; $25 \%$ de l'échantillon Fath sont ainsi des résidents de Naplouse, lesquels ne constituent que $18 \%$ de l'échantillon toutes tendances. Avec $34 \%$, les islamistes bénéficient également d'une forte surreprésentation dans l'échantillon des résidents de Naplouse qui ne constituent que $23 \%$ de l'échantillon toutes résidences ; les résidents de Naplouse constituent ainsi $27 \%$ de l'échantillon islamiste et $18 \%$ seulement de l'échantillon toutes tendances. La gauche, dans toutes ses composantes, connaît une forte sous-représentation (le PCP un peu moins que le FDLP et surtout le FPLP) : avec $42 \%$ de l'échantillon toutes résidences, la gauche ne constitue que $20 \%$ de l'échantillon des résidents de Naplouse ; ceux-ci, qui constituent $18 \%$ de l'échantillon toutes tendances, ne fournissent que $9 \%$ de l'échantillon de la gauche.

50 En abordant les régions Centre, les équilibres se renversent Ainsi le llwâ de Ramallâh se caractérise-t-il par une surreprésentation de la gauche due très largement au FDLP mais également au PCP, alors que le FPLP atteint tout juste la normale : avec seulement $42 \%$ de l'échantillon toutes résidences, la gauche constitue $55 \%$ de l'échantillon Ramallâh; avec $10 \%$ de l'échantillon toutes tendances, les résidents de Ramallâh constituent $14 \%$ de l'échantillon de la gauche. Ramallâh, en revanche, sous-représente légèrement le Fath : avec 33\% de l'échantillon toutes résidences, il ne constitue que $29 \%$ de l'échantillon Ramallâh; les résidents de Ramallâh, cependant, qui fournissent $10 \%$ de l'échantillon toutes tendances, constituent $9 \%$ de l'échantillon Fath. La sousreprésentation des islamistes, quant à elle, est beaucoup plus forte : avec $23 \%$ de l'échantillon toutes résidences, les islamistes ne constituent que $15 \%$ de l'échantillon Ramallâh; avec $10 \%$ de l'échantillon toutes tendances, les résidents de Ramallâh ne constituent que 7\% de l'échantillon islamiste.

51 Le llwâ de Jérusalem se caractérise par une très forte surreprésentation de la gauche, du FPLP tout particulièrement mais également du PCP alors que le FDLP ne parvient pas à atteindre la moitié de la normale: avec $42 \%$ seulement de l'échantillon toutes résidences, la gauche parvient à constituer $77 \%$ de l'échantillon de Jérusalem; à lui seul, le FPLP constitue $52 \%$ de l'échantillon de Jérusalem alors qu'il ne fournit que $22 \%$ de l'échantillon toutes résidences ; Jérusalem constitue ainsi $21 \%$ de l'échantillon de la gauche et $11 \%$ seulement de l'échantillon toutes tendances politiques. Le Fath, à l'inverse, paraît fortement sous-représente: avec 33\% de l'échantillon toutes résidences, il ne constitue que $11 \%$ de l'échantillon Jérusalem; les résidents de Jérusalem ne constituent ainsi que $4 \%$ de l'échantillon Fath alors qu'ils fournissent $11 \%$ de l'échantillon toutes tendances. Les islamistes, enfin, sont le plus massivement sousreprésentés à Jérusalem: avec $23 \%$ de l'échantillon toutes résidences, ils ne représentent que $4 \%$ de l'échantillon Jérusalem; les résidents de Jérusalem, qui constituent $11 \%$ de l'échantillon toutes tendances, ne fournissent ainsi que $2 \%$ de l'échantillon islamiste.

52 Le llwâ de Bethléem accorde lui aussi une surreprésentation à la gauche, au profit du FDLP (à la différence de Jérusalem) et du FPLP (dans une moindre mesure que Jérusalem) alors que la part du PCP parmi les résidents de Bethléem correspond exactement à celle qu'il tient parmi l'échantillon toutes résidences; la gauche, avec seulement $42 \%$ de l'échantillon toutes résidences, fournit $74 \%$ de l'échantillon Bethléem ; les résidents de Bethléem constituent ainsi $27 \%$ de l'échantillon de la gauche pour seulement $15 \%$ de l'échantillon toutes tendances. Comme à Jérusalem, mais dans une moindre mesure, le Fath est sous-représente à Bethléem : avec 33\% de l'échantillon 
toutes résidences, il ne constitue que 18\% de l'échantillon Bethléem; avec $15 \%$ de l'échantillon toutes tendances, les résidents de Bethléem constituent seulement $9 \%$ de l'échantillon Fath. Les islamistes, enfin, avec seulement $4 \%$ de l'échantillon Bethléem pour $23 \%$ de l'échantillon toutes résidences, sont considérablement sous-représentés ; les résidents de Bethléem, avec 15\% de l'échantillon toutes tendances, ne constituent ainsi' que $3 \%$ de l'échantillon islamiste.

Le llwâ d'Hébron accorde une surreprésentation au Fath, aux islamistes et au PCP tandis que les FPLP et FDLP s'y trouvent sous-représentés : avec 33\% de l'échantillon toutes résidences, le Fath constitue 39\% de l'échantillon Hébron; les résidents d'Hébron, $17 \%$ de l'échantillon toutes tendances, constituent ainsi $20 \%$ de l'échantillon Fath. Les islamistes, $23 \%$ de l'échantillon toutes résidences, constituent $27 \%$ de l'échantillon Hébron; les résidents d'Hébron, $17 \%$ de l'échantillon toutes tendances, constituent ainsi $20 \%$ de l'échantillon islamiste. La gauche en revanche est sousreprésentée, à l'exception du PCP qui constitue $11 \%$ de l'échantillon Hébron pour seulement $8 \%$ de l'échantillon toutes résidences. L'échantillon FPLP d'Hébron, en revanche, est pour moitié inférieur à sa part de l'échantillon toutes résidences ( $11 \%$ et $22 \%)$; le FDLP apparaît lui aussi sous-représenté, mais dans une moindre mesure : 5\% de l'échantillon Hébron pour $7 \%$ de l'échantillon toutes résidences; les résidents d'Hébron constituent ainsi $11 \%$ de l'échantillon FDLP pour 17\% de l'échantillon toutes tendances.

54 La Bande de Gaza, enfin, est la seule région à accorder une surreprésentation exclusive et massive aux islamistes : avec seulement $23 \%$ de l'échantillon toutes résidences, ils constituent 56\% de l'échantillon Gaza; Gaza, avec seulement 7\% de l'échantillon toutes tendances, constitue ainsi $27 \%$ de l'échantillon (islamiste. Les nationalistes, à l'inverse, se trouvent fortement sous-représentés : avec $76 \%$ de l'échantillon toutes résidences, ils ne constituent que $42 \%$ de l'échantillon Gaza; Gaza, avec $11 \%$ de l'échantillon toutes tendances ne constitue ainsi que $6 \%$ de l'échantillon nationaliste.

Type de résidence

55 Sont ici considérés comme citadins les résidents des 10 municipalités qui comptaient plus de 2000 électeurs inscrits lors des élections municipales de 1976 (Tûlkarm, Qalqîliyya, Jénine, Naplouse, al-Bîra, Ramallâh, Bethléem, Bayt Jâlâ, Bayt Sahûr et Jéricho) ainsi que Jérusalem et sa petite couronne (à l'exception bien entendu du camp de réfugiés de Qalandia). Ce critère, bien qu'arbitraire, se défend de deux points de vue : numériquement, tout d'abord, les municipalités ici considérées comme villes ont toutes plus de 12000 habitants ; "qualitativement» enfin, du fait du mode censitaire du scrutin municipal, il est donné à l'observateur de situer les concentrations particulièrement importantes de personnes à revenus situés au-dessus d'un certain seuil, concentrations qui désignent habituellement une ville. Du fait de leur caractère d'« étrangers » à la Cisjordanie, les résidents de la Bande de Gaza ont été exclus de ce traitement.

56 En l'absence de statistiques concernant les types de résidence de l'ensemble des effectifs universitaires, le seul élément de comparaison dont nous disposons est la répartition générale de la population de Cisjordanie entre citadins, ruraux et habitants des camps de réfugiés ${ }^{17}$. Le tableau 3 montre ainsi, au sein de l'échantillon élargi, une surreprésentation des citadins de plus de 10\% (50\% parmi les candidatures contre $38 \%$ parmi la population de Cisjordanie), au détriment des ruraux (41\% contre 54\%), les réfugiés des camps présentant des candidatures dans une proportion très légèrement 
supérieure à leur importance dans la population de Cisjordanie (9\% contre $8 \%)$. Cette distorsion, cependant, se trouve réduite au sein de l'échantillon des seuls élus ; si, avec $46 \%$ des élus, les ruraux sont encore en dessous de leur importance dans la population générale, les citadins doivent partager leur surreprésentation de l'échantillon élargi avec les réfugiés des camps, obtenant respectivement $43 \%$ et $12 \%$ des sièges. La forte présence des citadins est quasi intégralement due à l'université de Bethléem qui compte $67 \%$ de candidatures citadines (pour seulement $22 \%$ de candidatures présentées par des ruraux); si Bir Zeit sur représente légèrement les citadins, Polytechnique les sous-représente; Najâh et Hébron, enfin, s'accrochent sans s'en démaquer au taux de citadins observé parmi la population totale de Cisjordanie. L'image de la présence des ruraux par université est exactement le négatif de celle des citadins (sous- représentation massive à Bethléem, légère sous-représentation à Bir Zeit, légère surreprésentation à Polytechnique et représentation adéquate à Najâh et Hébron). La surreprésentation des réfugiés des camps parmi l'échantillon des élus est due principalement à Bethléem (17\%), à Polytechnique (13\%) et dans une moindre mesure Bir Zeit (9\%).

Comme il a été déjà souligné, l'échantillon citadin est marqué par un taux particulièrement important de femmes ( $20 \%$ face à $13 \%$ au sein de l'échantillon global) et de chrétiens ( $29 \%$ face à $16 \%$ dans l'échantillon global); les femmes se retrouvent ainsi à $77 \%$ citadines et les chrétiens le sont à $90 \%$. L'échantillon rural, quant à lui, se situe à l'exact opposé avec la participation féminine et chrétienne la plus faible ( $4 \%$ et $3 \%$ ), les femmes n'habitant les villages qu'à hauteur de $13 \%$ et les chrétiens à hauteur de $8 \%$ ). Avec ses $13 \%$ de candidatures présentées par des femmes, l'échantillon des réfugiés se situe à l'exacte moyenne tandis 'que la part de candidatures chrétiennes n'atteint que $4 \%$ de leurs effectifs. Face à de telles proportions, les chrétiens ne paraissent pas être lésés, leur présence dans les villages et les camps étant dérisoire. Les femmes, seules, sont systématiquement sous-représentées, en particulier à la campagne.

59 L'échantillon des citadins connaît une surreprésentation de candidats de gauche: l'échantillon de gauche compte en effet $63 \%$ de citadins, lesquels ne constituent que $50 \%$ de l'échantillon toutes tendances et $38 \%$ de la population générale ; avec seulement $45 \%$ de l'échantillon tous types de résidences, la gauche constitue ainsi $57 \%$ de l'échantillon citadin. Cette surreprésentation est principalement due au FPLP (66\% de citadins) et dans une moindre mesure aux PCP (63\%) et FDLP (57\%). La part de citadins chez les islamistes se situe au dessous de la moyenne si l'on considère l'échantillon élargi (44\% pour 50\%) mais au-dessus de la moyenne de l'échantillon des seuls élus (52\% pour $43 \%$ ). Avec seulement $32 \%$ de citadins, l'échantillon Fath, enfin, est de loin celui qui sous-représente cette catégorie de la population; bien que constituant $34 \%$ de l'échantillon tous types de résidence, le Fath ne fournit que $22 \%$ de l'échantillon citadin.

60 L'échantillon des ruraux apparaît comme le négatif de celui des citadins. Le Fath est l'échantillon dans lequel les ruraux sont le plus fortement surreprésentés avec 58\% contre $41 \%$ de moyenne; cette surreprésentation est surtout interne à l'échantillon universitaire élargi mais se réduit quelque peu si l'on considère la population générale de Cisjordanie dont les ruraux constituent encore 54\%; avec seulement $34 \%$ de l'échantillon tous types de résidences, le Fath constitue $47 \%$ de l'échantillon des ruraux. A l'inverse, avec seulement $27 \%$, les ruraux sont fortement sous-représentés dans 
l'échantillon de gauche; la gauche qui constitue $45 \%$ de l'échantillon tous types de résidence voit sa part descendre à $29 \%$ de l'échantillon des ruraux. Cette distorsion est presque exclusivement due au FPLP qui n'en compte que $19 \%$. Avec $48 \%$ de ruraux, les islamistes, enfin, sous-représentent légèrement cette catégorie si l'on considère la population générale $(54 \%)$ mais la surreprésentent par rapport à la moyenne universitaire élargie (41\%) ; l'échantillon des seuls élus renverse le rapport au sein des seuls échantillons universitaires avec $42 \%$ de ruraux parmi les islamistes pour $46 \%$ parmi l'échantillon toutes tendances et creuse la sous-représentation par rapport alla population générale.

61 Les réfugiés résidents des camps sont globalement légèrement sur-représentés, surtout dans l'échantillon des élus : avec $8 \%$ de la population, ils constituent $9 \%$ de l'échantillon élargi et $12 \%$ des élus. Cette sur-représentation est presque entièrement due au FPLP qui compte $16 \%$ de réfugiés des camps ainsi que - mais dans une moindre mesure - au Fath qui en compte $10 \%$. Les FDLP et PCP, au contraire, sous-représentent fortement cette catégorie de la population de même que les islamistes, dans une moindre mesure. Les facultés

62 La répartition des candidatures selon les facultés d'inscription laisse apparaître une identité propre à chaque université. Ainsi, si à Najâh c'est la faculté d'Économie qui est très fortement surreprésentée ( $40 \%$ de l'échantillon élargi pour seulement $26 \%$ des effectifs), à Bir Zeit c'est la faculté des Lettres qui jouit de ce privilège ( $72 \%$ pour $40 \%)$ au détriment des seules facultés des Sciences (6\% pour $23 \%$ ) et d'Ingénierie ( $4 \%$ pour 16\%). A Hébron, la faculté de Charîa est surreprésentée du fait des activités islamistes qui y sont quasi exclusivement concentrées ( $45 \%$ pour $32 \%$ ).

63 La ventilation des étudiants par faculté et par appartenance politique fait apparaître d'intéressantes identités : globalement, le Fath recrute ses candidats en tout premier lieu dans les facultés des Lettres et en second lieu dans les facultés d'Economie; les islamistes trouvent, quant à eux, leurs places fortes dans les facultés des Sciences, d'Ingénierie (deux facultés où la gauche se trouve quasiment inexistante) et d'Economie (à Najâh). A Hébron, le jeu est encore plus tranché puisque l'ensemble des candidats nationalistes proviennent de la faculté des Lettres tandis que $97 \%$ des islamistes sont inscrits dans la faculté de Charî’a. Curieusement, la faculté de Charî’a de Najâh ne fournit quasi aucune candidature, pas même islamiste.

Les appartenances politiques

64 Le Fath se caractérise ainsi par un taux de présence féminine et chrétienne inférieur à la moyenne de l'échantillon toutes tendances (caractéristique qu'il partage en second avec les islamistes) et par le taux le plus élevé de ruraux et le plus bas de citadins; il sur-représente très légèrement les réfugiés des camps. Le grand nombre de ses candidatures présentées par des résidents du Nord et du Sud recoupe des taux élevés de ruraux résidant dans ces régions et de quasi-absence de chrétiens. A l'inverse, les régions Centre à forte population citadine et chrétienne parmi laquelle les femmes manifestent un engagement politique important fournissent plutôt moins de candidatures Fath que la moyenne.

Le FPLP se situe à l'exact opposé du Fath avec le taux le plus bas de ruraux et le plus haut de citadins ; il se caractérise par le taux le plus élevé de femmes, de chrétiens et de réfugiés des camps. Le taux important de citadins y recoupe la forte présence féminine et chrétienne ainsi que celle des résidents des régions Centre. 

Le FDLP se situe à peu près dans la moyenne de la représentation féminine; chrétienne, d'enracinement : Ramallâh et Bethléem.

7 Après le FPLP, le PCP constitue la seconde organisation dans laquelle le taux de femmes, de chrétiens et de citadins est le plus élevé et le taux de ruraux le plus bas. A l'inverse du FPLP, cependant, il compte le taux le moins élevé de réfugiés des camps. Ses candidats sont concentrés dans les régions Centre mais également sud où ils constituent le premier groupe devant les islamistes et le Fath.

68 Les islamistes, enfin, se caractérisent par le taux le plus bas de femmes (de chrétiens bien évidemment) et de résidents des régions Centre. Ils proviennent massivement du Nord et de Gaza. Se situant à peu près à la moyenne de présence citadine et rurale, ils sous-représentent dans l'ensemble les réfugiés ${ }^{18}$.

Analyse des résultats des scrutins

9 L'étude des listes des candidats élus montre, pour le résultat de chaque scrutin, un très haut degré de cohésion politique et un nombre très faible de cas de "panachages " (rendus possibles par le non blocage des listes dans l'ensemble des établissements à l'exception de Bir Zeit); le choix se fait donc sur une base idéologique et non individuelle. La seule grande exception est l'élection à Najâh, en 1979, d'un communiste, isolé parmi dix islamistes : récemment libéré au terme de nombreuses années de prison, c'est son patriotisme qui est plébiscité et non pas son affiliation partisane.

70 Occasions périodiques de débattre publiquement des événements politiques du moment en termes d'affiliation partisane, les campagnes électorales ne paraissent pas déboucher sur une articulation directe à la scène politique palestinienne de "l'Extérieur » : la chronologie des alliances politiques pratiquées dans les 5 universités (figurées dans mes tableaux par une ou deux étoiles accolées aux résultats de chacun des membres des coalitions), ne montre que de très rares cas où des élections, dans un même trimestre, obéissent à un même schéma de coalitions, les particularités propres à chaque établissement l'emportant la plupart du temps sur des mots d'ordre extérieurs.

7 Cette absence d'articulation directe ne signifie évidemment pas que «l'Intérieur » demeure insensible à «l'Extérieur » : on remarque, par exemple, que la majorité des cas d'union nationale ont vu le jour avant l'éclatement de l'OLP en 1983, tandis qu'après cette date symbole, ce sont des listes d'union de la gauche qui se multiplient; de même, les menaces qui pèsent sur l'existence même de l'OLP après l'entrée de l'armée israélienne au Liban en 1982 entraînent-elles à Polytechnique, à Hébron et à Najâh un sursaut national (absent cependant à Bir Zeit et à Bethléem).

72 Si la volonté d'unité nationale demeure la priorité dans chaque négociation, la prise en compte des réalités "politiciennes" limite souvent l'unité à des alliances ponctuelles et restreintes à certaines organisations du camp nationaliste. A trois reprises seulement, le camp islamiste a accepté de retirer ses candidats au nom de l'impératif de l'unité nationale face à un camp nationaliste uni, entraînant ainsi l'annulation des élections au profit de la tazkiyya (à Hébron en 1982, à Najâh en 1983 et à Bethléem en 1986). L'unité nationale s'est faite à 10 reprises face aux islamistes (2 fois à Najâh, 2 fois à Bir Zeit, 1 fois à Bethléem, 3 fois à Hébron et à Polytechnique). L'absence d'unité même partielle à l'intérieur du camp nationaliste est un phénomène relativement rare 
puisque qu'il ne s'est produit qu'à 3 reprises (à Najâh en 1981 et en 1987, ainsi qu'à Polytechnique en 1987).

73 La présence d'une forte liste islamiste peut susciter une unité nationale de confrontation (par exemple Bir Zeit en 1979 et 1981) comme elle peut pousser le Fath à s'abstenir de toute alliance avec la gauche jugée moins nombreuse que les voix hésitant entre l'islam des « Frères » et celui du mouvement de Yasser Arafat (le cas de Najâh est particulièrement intéressant à l'exception des années 1980 et 1982). En certains cas, la volonté hégémonique du Fath empêche la mise en œuvre de listes d'union (en 1985, par exempte, le Fath réclame 5 des 9 sièges au conseil de Bir Zeit, conduisant la gauche à présenter une liste d'union face au Fath d'une part, et aux islamistes de l'autre). A Hébron, l'extrême faiblesse de la gauche, prise entre le Fath et les islamistes, explique sans aucun doute la propension des petites organisations marxistes à s'allier avec le Fath (avec une seule exception, en 1985, l'union de la gauche face aux deux « Grands » ne leur ayant rapporté que $6,5 \%$ des suffrages et aucun siège). Sur toute la période couverte, l'union de la gauche a pu se réaliser 9 fois ( 2 fois à Najâh en 1979 et 1986, à Bir Zeit en 1983 et 1985. à Bethléem en 1984 et 1985,1 fois à Hébron en 1985 et 2 fois à Polytechnique en 1983 et 1985). Ainsi que je l'ai souligné, c'est la période 1983-1986 qui a été le plus favorable ace type d'union. En dehors de ces schémas très clairs de coalitions, tout est possible: Bethléem, par exemple, offre les cas les plus « fantaisistes » les plus nombreux, variant d'une année à l'autre.

Najâh :

74 De façon générale, la scène politique estudiantine se caractérise par une bipolarité Fath-istamistes qui, selon les établissements, laisse plus ou moins d'« entre-deux » à la gauche. A Najâh (tableaux 5), cette bipolarité écrase la gauche: ayant toujours tait cavalier seul, les islamistes obtiennent un score qui tourne autour des $41 \%$, tandis que le Fath qui profite en 1980 et 1982 des suffrages de la gauche, obtient environ $49 \%$ des voix. Prise entre les deux, la gauche ne recueille qu'environ 10\% des suffrages (le score de 1979 doit être placé fout à fait à part, comme souligné plus haut). Lorsque les projets d'union nationale avec le Fath échouent, la gauche ne parvient qu'une seule fois à surmonter ses divisions internes. Face aux islamistes, pourtant, les nationalistes ont su présenter des listes d'union à 3 reprises. En 1981 comme en 1987, en revanche, aucun accord n'a pu être trouvé entre les diverses forces de l'OLP qui font chacune cavalier seul. Depuis janvier 1986, le Fath décide de faire cavalier seul, pensant- à tort si l'on considère ses scores - avoir à gagner des voix islamistes en refusant l'union avec la gauche. Le Fath de Najâh, d'ailleurs, a souvent fait l'objet de critiques de la part de la gauche qui lui reproche, face aux islamistes, une tiédeur qui, à l'occasion, se serait transformée en alliance.

75 Les islamistes, partis d'un très haut score en 1980 (plus de 59\% des voix), voient leurs résultats s'éroder pendant 2 ans, atteignant leur plus bas niveau en novembre 1982 avec seulement $35 \%$ des suffrages face à un camp nationaliste qui se ressaisit à la suite de l'invasion israélienne du Liban; ils remontent ensuite pour atteindre un nouveau palier situé aux alentours de $40 \%$ des suffrages.

Le mode majoritaire du scrutin, à l'évidence, favorise le Fath qui, de 1980 à 1987, a bénéficié de $61 \%$ des sièges pour seulement $49 \%$ des voix (grossies en 80 et 82 de celles de la gauche) ; les islamistes, quant à eux, n'ont obtenu que $21 \%$ des siéges pour $40 \%$ des suffrages ; cette disproportion s'accroît encore lorsqu'on considère que le Fath a obtenu $78 \%$ des présidences. Seule la gauche obtient un nombre de sièges à peu près 
proportionnel à ses scores en nombre de voix (encore faut-il remarquer qu'elle n'a jamais obtenu de sièges en dehors de listes d'union avec le Fath).

77 Najâh, en tout cas, est l'université qui, de 1978 à 1983, présente le plus de panachages, non seulement entre nationalistes mais également entre nationalistes et islamistes. Cela pourrait suggérer que, plus qu'ailleurs, la personnalité des candidats compte autant que leur affiliation politique (comme le cas de 1979 déjà souligné fa montré) ou encore que la ligne de démarcation entre le Fath et les islamistes n'est pas forcément perçue comme infranchissable.

Bir Zeit :

78 A Bir Zeit (tableaux 6), la précision des résultats dont nous disposons à partir de 1981 permet d'établir deux modes d'analyse des scrutins : le tableau 6.2 présente les scores obtenus par les têtes de listes; il constitue une hypothèse haute pour le Fath qui, dans 4 des 6 cas étudiés, se voit attribuer les voix de plusieurs ou même de l'ensemble des organisations de gauche dans le cadre des accords de coalition. Le tableau 6.3 établit, quant à lui, le score théorique de chaque organisation membre d'une coalition par la division par 9 des résultats additionnés de ses candidats; il constitue une hypothèse basse pour le Fath qui se voit retirer les voix obtenues par les candidats de gauche dans le cadre de coalitions dont il est le moteur (le FDLP se trouve légèrement désavantagé dans les totaux du fait de son boycott des élections de 1983). Le tableau 6.1, en donnant le nombre de candidats élus par appartenance politique, donne une idée intéressante de la perception des rapports de force par l'ensemble des groupes membres d'une coalition, le nombre de sièges laissés à chaque groupe étant négocié lors des accords de coalition au prorata de la représentativité supposée de chacun.

79 Si l'on considère l'hypothèse basse, on s'aperçoit que Fath et islamistes se situent à peu près à égalité avec $31 \%$ des voix, bipolarité qui se trouve déstabilisée par une gauche qui, à elle seule et de manière théorique, rassemble plus de voix que chacune des deux grandes organisations. Ce rapport de force, tout à fait particulier à Bir Zeit, a conduit à l'établissement de nombreuses listes d'union partielle, l'unité nationale totale n'étant atteinte qu'une seule fois en 1981; même les événements du Liban en 1982-1983 ne parviennent pas à réitérer une telle union.

Comme à Najâh, les islamistes partent d'un score très élevé, avec $43 \%$ des suffrages en 1979, connaissent ensuite un tassement au-dessous de la barre des $30 . \%$, pour remonter à 34,3\% des suffrages en janvier 1987.

81 Le mode majoritaire de scrutin, plus encore qu'à Najâh à cause du blocage des listes, favorise le Fath et lamine complètement les islamistes: alors que dans l'hypothèse basse, le Fath n'obtient que 31,2\% des voix et que dans l'hypothèse haute il en obtient $46 \%$, au moins $60,3 \%$ des sièges lui reviennent et $100 \%$ des présidences. Avec leur $31 \%$ environ des suffrages, les islamistes n'ont jamais obtenu de sièges. La représentation de la gauche apparaît, quant à elle, très diversifiée : le FPLP n'obtient que 6 sièges entre 1981 et 1987 soit $11,5 \%$ pour un score (hypothèse haute) de 20,2\%. Le PCP et le FDLP, en revanche, peuvent être satisfaits d'une quasi-adéquation entre les suffrages obtenus et la part de représentants élus.

Bethléem :

82 L'université de Bethléem (tableaux 7) constitue une exception remarquable parmi toutes les universités de Cisjordanie en accordant $90 \%$ de ses suffrages à la gauche aux élections "administratives " (et près de $80 \%$ aux élections du conseil plénier et des clubs). Cette gauche est de très loin dominée par le FPLP qui emporte $42,5 \%$ des 
suffrages, suivi du PCP avec 27,5\% et enfin du FDLP avec 17,5\% des voix. Par le jeu des élections ouvertes aux seuls membres des clubs qui permet un investissement aussi massif que sélectif des organisations minoritaires, les partisans du Fath sont parvenus à obtenir 20\% de représentants au sein du conseil tandis que les islamistes, en 1985 et en 1987, ont envoyé l'un des leurs au conseil. Ainsi que je l'ai déjà fait remarquer, les schémas de coalitions, très nombreuses à Bethléem, ont été des plus divers n'obéissant la plupart du temps qu'aux règles de la " politique politicienne ». Le PCP n'a jamais fait cavalier seul tandis que le FDLP n'a qu'une seule fois tenté seul sa chance (abandonnant même la partie en 1985) ; le FPLP, quant à lui, à quatre reprises, a préféré compter ses voix. L'union nationale n'a été réalisée qu'en octobre 1986, après la dénonciation de l'accord jordano-palestinien, tandis qu'un front arafatiste est constitué en 1987 face au FPLP, qui maintient son refus d'adopter les résolutions de CONU pour base d'un règlement.

L'implantation du Fath est relativement récente à Bethléem, retardée par la force des partisans du Front du refus : les chiffres de 1987 montrent bien cette réticence vis-à-vis de Yasser Arafat, près de $41 \%$ des surnages allant au seul FPLP (et 16\% aux islamistes partisans de la Grande Palestine) face au $43 \%$ des voix accordées à la coalition Fath, FDLP et PCP. Tard venus sur la scène de Bethléem marquée par le nationalisme de gauche et l'engagement des chrétiens, les islamistes ont créé la surprise en obtenant $11 \%$ des suffrages en 85 . S'étant retirés de la compétition en 86 pour laisser place à un processus de tazkiyya, ils gagnent comme partout ailleurs de nombreuses voix en 1987. Hébron :

84 La pauvreté des résultats chiffrés disponibles pour les élections de l'université d'Hébron empêche toute analyse détaillée (tableaux 8). Avec 48\% d'élus Frères musulmans, l'université islamique se place en tête des universités de Cisjordanie pour la présence des islamistes. Avec $42,6 \%$ des suffrages, le Fath ne constitue que la seconde force politique sur le campus tandis que la gauche est réduite à quelque $8 \%$ des voix. Face aux puissants islamistes, les organisations nationalistes ont toujours essayé de faire front commun et à 3 reprises sont parvenues à constituer des coalitions d'unité nationale et à 2 reprises des listes d'union de la gauche.

Polytechnique :

Polytechnique (tableaux 9) présente également une bi-polarité entre le Fath qui, entre 1982 et 1987, obtient la majorité absolue des voix et les islamistes qui obtiennent $41 \%$ des suffrages, la gauche se partageant les miettes restantes. Malgré cette forte présence proportionnelle durant cette période, les islamistes n'obtiennent qu'un seul élu. En 1981, ils emportent l'ensemble des sièges du conseil (mais les résultats chiffrés ne sont pas disponibles). Le FDLP n'est jamais parvenu à présenter de candidat tandis que la gauche a toujours constitué des listes d'union (parfois avec le Fath). Seules les élections de 1987 ont vu la concurrence de 5 listes distinctes. Comme ailleurs, les islamistes partent d'un score élevé en 1982 pour connaître ensuite une décrue puis une remontée amorcée seulement en 1987.

Rapportés à la sociologie du leadership de "l'Intérieur » que j'avais dressée l'an dernier, nous pouvons conclure au caractère prémonitoire des tendances dessinées par les élections étudiantes depuis 1978 : l'engagement politique des femmes, la prise en main de leur destin par les ruraux, l'activisme débordant des réfugiés, la contribution essentielle des populations du Nord et de la Bande de Gaza à la détermination d'une ligne politique, la capacité mobilisatrice des islamistes sont autant de phénomènes dont 
la force s'est développée ces dix dernières années sur les campus et qui se sont soudainement manifestés avec le soulèvement comme des caractéristiques désormais étendues à l'ensemble de la société. Il ne fait aucun doute que c'est l'université qui a constitué l'un des principaux outils de l'intégration de ces catégories traditionnellement marginalisées au sein du leadership de "l'Intérieur»: sous la pression de la mobilisation populaire, ces "marginaux", dont bon nombre avaient milité dans les conseils étudiants, se sont retrouvés de facto inclus dans les organes de décision (structurés ou non) du soulèvement, au moins dans un premier temps.

Des questions se posent cependant sur la capacité des diverses forces de l'échiquier politique à intégrer ces nouvelles données. Est-il possible d'affirmer, par exemple, que le soulèvement, sous certains aspects, constitue une sorte de "coup d'État " contre l'establishment politique de "l'Intérieur» dont le processus de confiscation, par les élites citadines et intellectuelles de la conurbation de Jérusalem regroupées au sein du Fath, parvenait à son acmé ? Durant la décennie 80 , on aurait été ainsi en présence de deux phénomènes parallèles et contradictoires : sur les campus comme " en ville ", la «palestinisation » des idéologies politiques (et religieuses) parvenait à renverser quasi totalement le panarabisme des années $70^{19}$ et s'imposait à terme à l'OLP qui avait, dés le milieu des années 70 , réellement gagné la représentativité majoritaire de «l'Intérieur »; mais alors que ce processus se traduisait « en ville » par la montée des couches urbaines liées à l'OLP (et tout particulièrement au Fath) massivement regroupées dans la conurbation de Jérusalem et dans les professions « intellectuelles ", les campus donnaient la parole aux militants de la même OLP mais dont beaucoup étaient originaires des camps et des campagnes, du Nord et du Sud, dans une large diversité organisationnelle que le système électoral cherchait à contenir ; les islamistes, de leur côté et dès le début des années 80 , venus du Nord, du Sud et de la Bande de Gaza, tentaient de trouver une place au sein de l'échiquier politique sans y parvenir vraiment. Durant toute cette période, on se trouvait donc au sein du Fath majoritaire devant une contradiction importante entre un leadership citadin de la conurbation de Jérusalem et une base plutôt rurale du Nord et du Sud.

88 Avec le soulèvement, le rôle des campagnes, du Nord (notamment Naplouse), de la Bande de Gaza et des camps dans la mobilisation populaire est apparu dans toute sa force. Le leadership institutionnel "national», en distinction des leaderships régionaux et locaux, a-t-il suivi ? Là est la question. Si l'on considère le camp nationaliste, nombreux sont les indices qui montrent une incapacité à intégrer les non "citadins de la conurbation de Jérusalem »; incapacité matérielle liée à la distance géographique et aux problèmes de sécurité, ou défense d'un quasi-monopole, la question est posée. Face aux islamistes, en revanche, les nationalistes se sont trouvés contraints de mener une certaine ouverture : la crise de l'été 1988 entre le CNU et Hamas a débouché sur une sorte de "reconnaissance ", de la part de l'OLP, d'une certaine légitimité islamiste à œuvrer sur la scène politique de «l'Intérieur " ${ }^{20}$.

Les événements de cette dernière année (1990) auraient tendance à mettre en évidence ces problèmes d'intégration de catégories jusque-là marginalisées à la scène politique nationale, toujours animée par les résidents de la conurbation de Jérusalem. Au sein du camp nationaliste, d'une part, des tensions entre Naplouse et le CNU par exemple sont apparues à diverses reprises: les mises en garde du Commandement sur la multiplication des éliminations de « collaborateurs » ont été battues en brèche tant par les Aigles Noirs que par les Panthères Rouges liées au Fath et au FPLP de la région ; à la 
veille de la fête de clôture du Ramadan, c'est l'ensemble des comités populaires de la ville qui rejettent l'appel à la grève générale lancé par le $\mathrm{CNU}$ et décident de ne pas diffuser son communiqué national. La Bande de Gaza semble également demeurer dans son isolement des deux dernières décennies et sa spécificité n'apparaît vraiment qu'à travers les mouvements du Jihâd islamique et de Hamas.

Les relations entre nationalistes et islamistes se sont également dégradées ces derniers mois et les heurts actuels entre Hamas et Fath dans le Nord (Naplouse, Jénine et camp de Tûlkarm) comme à Gaza (camps de Jabalyâ et de Burayj) laissent présager du pire : après avoir acquis la reconnaissance de leur place sur l'échiquier intérieur mais avoir échoué à obtenir une importante représentation au sein du CNP, les Frères musulmans apparaissent décidés à prendre d'assaut la citadelle du leadership de "l'Intérieur ", jusque-là monopolisé par les nationalistes.

91 La réouverture, en octobre 90, de l'université de Bethléem devrait être suivie de celle des autres universités de Cisjordanie ; il sera alors intéressant d'analyser les résultats des élections aux conseils étudiants qui s'y tiendront pour avoir une' idée, trois années après le début du soulèvement, des éventuelles continuités ou des renversements sur l'échiquier politique (renforcement des islamistes, contrôle accru du camp nationaliste par les réfugiés et les ruraux ?). Les toutes dernières élections tenues à Gaza au sein des employés de l'UNRWA (Organisation -des Nations Unies pour les Réfugiés), en tous cas, laissent présager un net renforcement des islamistes; en sera-t-il de même en Cisjordanie?

\section{NOTES}

1.Cette concurrence a fait l'objet de mon intervention au premier colloque francoégyptien de politologie, "Récentes transformations politiques dans le monde arabe ", Le Caire 15-18 Janvier 1988; cf. « Le pouvoir en Cisjordanie : un combat à quatre », Bulletin du CEDEJ 23, $1^{\text {er }}$ semestre 1988, p.149-182.

2.Voir mon article "L'Intifâda dans sa troisième année ", Esprit, 163, juillet-août 1990, p.15-21.

3.Paris 3-5 juillet 1989, « Le leadership palestinien de l'intérieur (Document Husaynî, été 1988) ", in Etudes politiques du monde arabe. Approches globales et approches spécifiques, CEDEJ, Le Caire 1991 et Egypte/Monde arabe n³, 3e trimestre 1990, p.121.

4.Les chiffres proviennent des annuaires du Conseil de l'Enseignement Supérieur. 5.La Cisjordanie compte également la petite université de Jérusalem qui dépend de l'administration des Biens religieux ainsi que des instituts professionnels. Voir, entre autres ouvrages, Majlis al-Ta'lîm al-'Alî (Conseil de l'Enseignement Supérieur), Dalîl alJâmi'ât al-Filastiniyya (Guide des universités palestiniennes), s. 1., 1985 ; Salâh AL-ZARU, al-Ta'lîm al-'Alî fî-l-Arâdî al-Muhtalla (L'enseignement supérieur dans les territoires occupés), Râbitat al-Jâmi'iyyîn, Hébron 1989 ; Samir N. Anabtawi, Palestinian higher education in the West Bank and Gaza. A critical assessment, KPI, Londres 1986. 
6. Muhammad Chadid, cité in Kate Rouhana, « Universities' ballot box gives students needed voice », al-Fajr Weekly, février 1986. Voir également As'ad 'Abd Al-Rahman, « Kharîtat al-Tayyârât wa-l-'Alâqât al.Siyâslyya al-Tâlibiyya fî-l-Jâmi'ât li-l-Manâtiq alFilastiniyya al-Muhtalla » (Géographie des tendances et des relations politiques estudiantines dans les universités des territoires palestiniens occupés), al-Qabas (Koweït), 29 avril 1985.

7.Kate Rouhana, op. cit.

8.Je tiens à remercier ici tous ceux qui ont bien voulu répondre à mes questions : à Najâh, Musaddiq al-Masrî, élu sur une liste Chabîba en 86, devenu en 1987 adjoint au responsable des relations publiques de l'université, Husâm al-Charif élu sur la liste islamiste en 78 et 79, ainsi qu'un étudiant Chabîba de la fin des années 70 ; à Bir Zeit, Ghassan Faramand, Dean of students, un membre de son bureau et Marwân al-Barghûthî, président Chabîba du conseil en 87, maintenant exilé à Amman ; à Bethléem, Manuel Hassassian, Dean of students et Muhammad al-Munâsira, élu Taqaddum en 83 et 84 ; à l'université islamique d'Hébron, Imràn al-Tamîmî, président de l'Association des universitaires et Mahmûd Faqûsa, vice-président Chabîba en 85 ; à Polytechnique. Ahmad Ribi président Chabîba en 84 et 85. D'anciens étudiants ou responsables politiques m'ont également aidé pour tel ou tel cas. La documentation du Centre d'études arabes de Jérusalem (Fayçal Husaynî) et de Dâr al-Jalîl à Amman, ainsi que les dossiers personnels d'Abd al-Jawâd Sâlih, maire d'al-Bira exilé à Amman, m'ont également été d'un grand secours.

9.Avec une part de 51\% de l'échantillon élargi, le seul échantillon des élus ne se situe pas dans une proportion telle qu'il infléchirait les tendances manifestées par l'échantillon élargi vers les siennes propres.

10.Aucune étude ne peut être exhaustive en ce domaine, les tribunaux militaires, pas plus que l'administration militaire ou civile, ne publiant d'état de leurs décisions. Il faut donc avoir recours à la presse et aux enquêtes menées par les divers centres, israéliens et palestiniens, de défense des droits de l'homme, al-Haq, Law in the Service of Man, Ramallâh ; Palestine Human Rights Information Center, Jérusalem et Chicago ; Betselem, The Israël Information Center for Human Rights in the Occupied Territories, Jérusalem. Les bulletins des universités publient parfois la liste de leurs étudiants victimes de la répression.

11.La répression semble s'exercer proportionnellement de façon plus importante contre les résidents de la région de Ramallâh et du Nord (Jénine et Tûlkarm) qu'à l'encontre des résidents du Sud, contre les réfugiés des camps et les villageois qu'à l'encontre des citadins, contre les musulmans qu'à rencontre des chrétiens, contre les élus de Bir Zeit qu'à l'encontre des étudiants des autres universités mais indistinctement contre les hommes et les femmes. Les islamistes n'ont été que très rarement victimes de la répression, contrairement aux militants du Fath (peut-être parce que plus souvent élus ?).

12. Cette proportion est tout particulièrement importante pour une population arabe. Il semble cependant qu'en ce qui concerne les effectifs étudiants envoyés dans les universités étrangères, le taux de femmes y soit quasiment nul.

13.Cette disproportion se réduit de façon constante: en 1980, l'institut comptait 3 étudiantes pour 222 étudiantes ; en 1986, il compte 79 étudiantes pour 918 étudiants. 14.Les chiffres concernant la répartition confessionnelle cités dans les tableaux proviennent de Daphne Tsimhoni, « Between the hammer and the anvil : the national 
dilemna of the Christian minority in Jerusalem and the West Bank », Orient, décembre 1983, p. 637-644. A Gaza, les chrétiens sont statistiquement quasi négligeables.

15.AFP, 25 mai 1987.

16.Les chiffres concernant la population de Cisjordanie cités dans le tableau proviennent d'un document inédit de l'ex-ministère jordanien des Affaires des Territoires occupés. Direction des Affaires municipales.

17.Les chiffres concernant la population globale ont pour base les données publiées in Meron Benvenisti et Shlomo Khayat, The West Bank and Gaza Atlas, West Bank Data Base Project, Jérusalem 1988, auxquelles j'ai appliqué mes propres critères de classification. 18.II ne faut pas oublier que l'échantillon ici concerné exclut les résidents de Gaza. 19.Les derniers développements avec la crise du Golfe et le soutien unanime accordé dans les territoires occupés à Saddam Hussein, le nouveau libérateur attendu de la Palestine, montre que le phénomène n'était pas irrémédiable: d'une période durant laquelle les Palestiniens étaient décidés à prendre en main la défense de leur destin, nous sommes, semble-t-il, retournés à une période d'espoir de libération venue de l'extérieur.

20.Cf. Jean-François Legrain, « Mobilisation islamiste et soulèvement palestinien, 1987-1988 », in Gilles Kepel et Yann Richard, Intellectuels et militants de l'islam contemporain, Le Seuil, Paris 1990, p. 131-166.

\title{
INDEX
}

Mots-clés : démocratie, démocratisation, Palestine, élections, Cisjordanie, OLP

\author{
AUTEUR \\ JEAN-FRANÇOIS LEGRAIN \\ CEDEJ-CNRS
}

